\title{
Esculetin suppresses lipopolysaccharide-induced inflammatory mediators and cytokines by inhibiting nuclear factor- $\kappa B$ translocation in RAW 264.7 macrophages
}

\author{
SU HYUN HONG ${ }^{1}$, HUI-KYUNG JEONG ${ }^{1}$, MIN HO HAN ${ }^{1,2}$, CHEOL PARK $^{3}$ and YUNG HYUN CHOI ${ }^{1,2}$ \\ ${ }^{1}$ Department of Biochemistry, Dongeui University College of Oriental Medicine, Busan 614-052; \\ ${ }^{2}$ Anti-Aging Research Center and Blue-Bio Industry and ${ }^{3}$ Department of Molecular Biology, \\ College of Natural Sciences, Dongeui University, Busan 614-714, Republic of Korea
}

Received January 7, 2014; Accepted October 2, 2014

DOI: $10.3892 / \mathrm{mmr} .2014 .2613$

\begin{abstract}
Although previous studies have demonstrated that the natural coumarin compound esculetin possesses various pharmacological properties, the molecular mechanism of esculetin-mediated anti-inflammatory potential is not fully understood. In this study, we determined the effects of esculetin on lipopolysaccharide (LPS)-induced inflammatory responses of murine RAW 264.7 macrophages. The results indicate that esculetin inhibits LPS-induced nitric oxide and prostaglandin $\mathrm{E}_{2}$ production in a concentration-dependent manner, and inhibits inducible nitric oxide synthase and cyclooxygenase-2 expression in RAW 264.7 cells. Esculetin also significantly suppresses the production of inflammatory cytokines, including tumor necrosis factor- $\alpha$ and interleukin-1 $\beta$, which was concomitant with a decrease in their expression levels. Furthermore, it was observed that esculetin attenuated the LPS-mediated nuclear factorkappa $\mathrm{B}(\mathrm{NF}-\kappa \mathrm{B})$ translocation associated with the blocking of inhibitor of NF- $\kappa \mathrm{B}(\mathrm{I} \kappa \mathrm{B})-\alpha$ degradation as well as reactive oxygen species (ROS) production, without any significant cytotoxicity. These data suggest that, by blocking NF- $\kappa \mathrm{B}$ activation, esculetin suppresses LPS-elicited inflammatory events, and this is mediated, at least in part, by inhibiting the generation of ROS. Collectively, these findings provide mechanistic insights into the anti-inflammatory action of esculetin in macrophages.
\end{abstract}

\section{Introduction}

Inflammation is a complex physiological and pathological process accompanied by the activation of the immune system

Correspondence to: Professor Yung Hyun Choi, Department of Biochemistry, Dongeui University College of Oriental Medicine, San 45-1, Yangjung-dong, Busanjin-gu, Busan 614-052, Republic of Korea

E-mail: choiyh@deu.ac.kr

Key words: esculetin, RAW 264.7 macrophages, inflammation, nuclear factor-kappa B, reactive oxygen species following foreign invasion or tissue damage. Although acute inflammation is a part of the defense response by organisms to remove injurious stimuli, chronic inflammation is associated with a wide variety of diseases, including cardiovascular, autoimmune and pulmonary diseases, Alzheimer's disease, diabetes, arthritis and cancer (1-3). Macrophages are key players in the immune response to foreign invaders, including infectious microorganisms. However, macrophages activated during chronic inflammation play an essential role in inflammation-related diseases through excessive production of inflammatory mediators, nitric oxide (NO) and prostaglandin $\mathrm{E}_{2}\left(\mathrm{PGE}_{2}\right)$, reactive oxygen species (ROS) and other inflammatory cytokines including tumor necrosis factor- $\alpha$ (TNF- $\alpha$ ) and interleukin-1 $\beta$ (IL-1 $\beta)(1,4,5)$.

Nuclear factor-kappa B $(\mathrm{NF}-\kappa \mathrm{B})$ is a transcription factor that plays a central role in the onset of inflammation, which normally presents in the cytosol and exists as an inactive complex with a class of inhibitory proteins, known as inhibitor of $\mathrm{NF}-\kappa \mathrm{B}(\mathrm{I} \kappa \mathrm{B})$ proteins. The NF- $\kappa \mathrm{B}$ dimer dissociates from $\mathrm{I} \kappa \mathrm{B}$ and translocates to the nucleus following an inflammatory stimulus, where it binds to promoter regions and induces the expression of a wide variety of genes involved in inflammation $(6,7)$. Due to its ubiquitous role in the pathogenesis of inflammatory gene expression, treatments aimed at inhibiting $\mathrm{NF}-\kappa \mathrm{B}$ may have potential therapeutic advantages in inflammatory diseases. In addition, the generation of ROS by activated macrophages initiates cascades of pro-inflammatory reactions. Since overproduction of ROS by macrophages causes oxidative damage to membrane lipids, DNA, proteins and lipoproteins, the excessive generation of intracellular ROS is one of the most notable hallmarks in the inflammatory process. A number of previous studies have demonstrated that ROS participate in the modulation of NF- $\kappa \mathrm{B}$ activation and that a broad range of antioxidants also abolish NF- $\kappa \mathrm{B}$ activation and intracellular ROS generation $(8,9)$. These observations indicate that the redox status of cells participates in modulating $N F-\kappa B$ activation $(10,11)$. Therefore, inhibition of ROS production associated with inactivation of $\mathrm{NF}-\kappa \mathrm{B}$ is a common therapeutic target for numerous inflammatory diseases.

Recently, natural medicinal plants as sources of natural products have been further developed for use in functional 
health foods and are typically used for preventing disease rather than curing it, or promoting health. Coumarin compounds are the major components of medicinal plants and are attracting interest due to their beneficial effects on human health (12). Among them, esculetin is a natural coumarin derivative identified in various medicinal plants used as folk medicines. This compound has multiple benefits, including anti-inflammatory $(13,14)$, antioxidative $(15-17)$, neuro-protective (18), immunomodulatory (19) and antitumor activities $(20,21)$. However, the detailed molecular anti-inflammatory mechanism of esculetin has not yet been studied. In the present study, we demonstrate for the first time that esculetin inhibits the production of inflammatory mediators and cytokines as well as ROS generation via the inhibition of the NF- $\kappa$ B activation pathway in lipopolysaccharide (LPS)-stimulated RAW 264.7 macrophages. The findings suggest that esculetin has therapeutic potential against inflammatory diseases.

\section{Materials and methods}

Materials. LPS (Escherichia coli 026:B6), Griess reagent, 2',7'-dichlorodihydrofluorescein diacetate (DCFH-DA) and 3-(4,5-dimethylthiazol-2-yl)-2,5-diphenyltetrazolium bromide (MTT) were purchased from Sigma-Aldrich (St. Louis, MO, USA). Dulbecco's modified Eagle's medium (DMEM), penicillin, streptomycin and fetal bovine serum (FBS) were obtained from Gibco-BRL (Gaithersburg, MD, USA). Specific antibodies against inducible nitric oxide synthase (iNOS), cyclooxygenase-2 (COX-2), TNF- $\alpha$, IL-1 $\beta, \mathrm{NF}-\kappa \mathrm{B}$ p65 and I $\mathrm{B}-\alpha$ were purchased from Santa Cruz Biotechnology, Inc. (Santa Cruz, CA, USA). Antibodies against nucleolin and actin were purchased from Cell Signaling Technology (Beverly, MA, USA). Esculetin (6,7-dihydroxycoumarin, 98\% purity, Fig. 1) was purchased from Sigma-Aldrich and dissolved in dimethyl sulfoxide (vehicle). All other chemicals not specifically cited were purchased from Sigma-Aldrich.

Cell culture and cell viability assay. The murine macrophage cell line RAW 264.7 was obtained from the American Type Culture Collection (Manassas, VA, USA) and cultured in DMEM supplemented with 10\% FBS, 2 mM L-glutamine, $100 \mathrm{U} / \mathrm{ml}$ penicillin and $100 \mathrm{~g} / \mathrm{ml}$ streptomycin at $37^{\circ} \mathrm{C}$ in a $5 \% \mathrm{CO}_{2}$ humidified air environment. Cell viability was evaluated using the MTT assay, which is based on the conversion of MTT to MTT-formazan by mitochondria.

Nitrite measurement. RAW 264.7 macrophages were plated in 24-well plates at $5 \times 10^{5}$ cells/well, and treated with various concentrations of esculetin in the absence or presence of $500 \mathrm{ng} / \mathrm{ml}$ LPS. Twenty-four hours later, the supernatant of cell cultures was collected, mixed with an equal volume of Griess reagent and then incubated at room temperature for $10 \mathrm{~min}$. $\mathrm{NaNO}_{2}$ was used to generate a standard curve, and nitrite production was determined by measuring optical density at $550 \mathrm{~nm}(22)$.

Enzyme-linked immunosorbent assay (ELISA). The levels of $\mathrm{PGE}_{2}$, TNF- $\alpha$ and IL- $1 \beta$ were determined by ELISA. ELISA kits from R\&D Systems (Minneapolis, MN, USA) were employed for the measurement of TNF- $\alpha$ and IL-1 $\beta$, and a kit

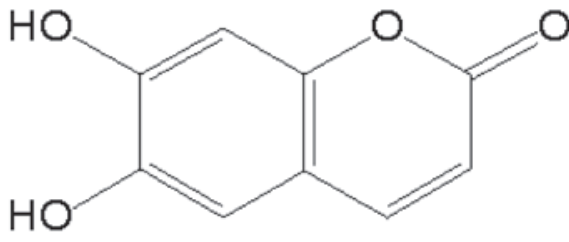

Figure 1. Chemical structure of esculetin.

from Cayman Chemical (Ann Arbor, MI, USA) was employed for the measurement of $\mathrm{PGE}_{2}$ (23).

Western blot analysis. The total cellular proteins were extracted with lysis buffer $(20 \mathrm{mM}$ sucrose, $1 \mathrm{mM}$ EDTA, $20 \mu \mathrm{M}$ Tris-1, pH 7.2, $1 \mathrm{mM}$ DTT, $10 \mathrm{mM} \mathrm{KCl,} 1.5 \mathrm{mM} \mathrm{MgCl} \mathrm{m}_{2}$ and $5 \mu \mathrm{g} / \mathrm{ml}$ aprotinin) for $30 \mathrm{~min}$. In a parallel experiment, nuclear and cytosolic proteins were prepared using nuclear extraction reagents (Pierce, Rockford, IL, USA) according to the manufacturer's instructions. Equal amounts of protein were separated on SDS-polyacrylamide gels and transferred to nitrocellulose membranes (Schleicher \& Schuell, Keene, $\mathrm{NH}$, USA) by electroblotting. After $2 \mathrm{~h}$ blocking with $5 \%(\mathrm{w} / \mathrm{v})$ nonfat milk in TBST (1.5 M NaCl, $20 \mathrm{mM}$ Tris- $\mathrm{Cl}, 0.05 \%$ (v/v) Tween-20, pH 7.4), the membranes were incubated overnight at $4^{\circ} \mathrm{C}$ with the desired antibodies. The blots were then washed with TBST $2 \mathrm{~h}$ prior to incubation at room temperature with peroxidase-conjugated secondary antibodies, and immunoreactive bands were visualized using an enhanced chemiluminescence detection system (Amersham Corp., Arlington Heights, IL, USA).

Measurement of ROS generation. To measure intracellular ROS, the cells were incubated for $4 \mathrm{~h}$ at $37^{\circ} \mathrm{C}$ in phosphate-buffered saline containing $20 \mathrm{mM}$ DCFH-DA to label intracellular ROS. The ROS production in the cells was monitored with a flow cytometer (FACSCalibur; Becton Dickinson, San Jose, CA, USA) using CellQuest Pro software (Becton Dickinson).

Statistical analysis. The data are expressed as the means \pm standard deviation. Statistical comparisons were performed using SPSS 12.0 (SPSS, Inc., Chicago, IL, USA) followed by Fisher's test. Significant differences between the groups were determined using the unpaired Student's t-test. $\mathrm{P}<0.05$ was considered to indicate a statistically significant result.

\section{Results}

Esculetin inhibits the production of pro-inflammatory mediators in RAW 264.7 macrophages. In order to test whether esculetin was capable of reversing LPS-induced accumulation of $\mathrm{NO}$ and $\mathrm{PGE}_{2}$, RAW 264.7 macrophages were treated with various concentrations of esculetin for $1 \mathrm{~h}$ before being stimulated with LPS for $24 \mathrm{~h}$. As demonstrated in Fig. 2A and B, treatment of RAW 264.7 cells with LPS caused a marked increase in the production of $\mathrm{NO}$ and $\mathrm{PGE}_{2}$. When the cells were incubated with esculetin $(12 \mu \mathrm{g} / \mathrm{ml})$ alone, the concentration of $\mathrm{NO}$ and $\mathrm{PGE}_{2}$ remained at a background level similar to that in the unstimulated culture. However, treatment with 

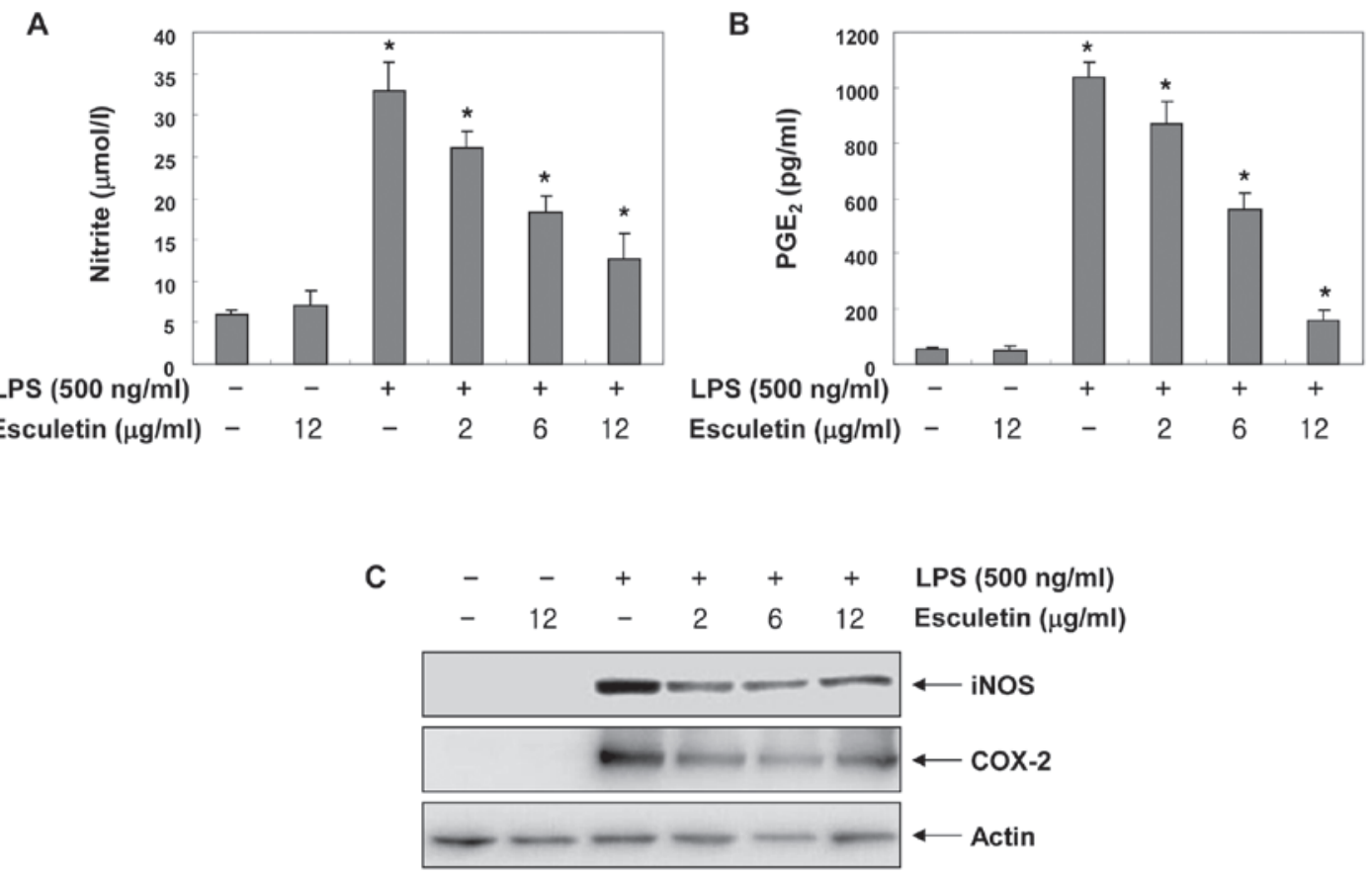

Figure 2. Inhibition of nitric oxide (NO) and prostaglandin $\mathrm{E}_{2}\left(\mathrm{PGE}_{2}\right)$ production, and inducible nitric oxide synthase (iNOS) and cyclooxygenase-2 (COX-2) expression by esculetin in lipopolysaccharide (LPS)-stimulated RAW 264.7 macrophages. RAW 264.7 cells were pre-treated with various concentrations of esculetin for $1 \mathrm{~h}$ prior to incubation with LPS $(500 \mathrm{ng} / \mathrm{ml})$ for $24 \mathrm{~h}$. Nitrite content was measured using the Griess reaction (A) and PGE ${ }_{2}$ concentration was measured in culture media using a commercial enzyme-linked immunosorbent assay kit (B). Each value indicates the mean \pm standard deviation and is representative of results obtained from three independent experiments. " $\mathrm{P}<0.05$ compared with cells treated with LPS in the absence of esculetin. (C) Cell lysates were prepared from cells grown under the same conditions and western blot analysis was performed using antibodies specific to murine iNOS and COX-2, and an enhanced chemiluminescence detection system. Actin was used as an internal control.

esculetin for $1 \mathrm{~h}$ prior to being incubated with LPS resulted in a dose-dependent inhibition of the LPS-induced $\mathrm{NO}$ and $\mathrm{PGE}_{2}$ production in RAW 264.7 cells.

Esculetin inhibits the expression of iNOS and COX-2 in RAW 264.7 macrophages. To determine whether esculetin decreases $\mathrm{NO}$ and $\mathrm{PGE}_{2}$ production via the suppression of iNOS and COX-2 expression, the levels of iNOS and COX-2 proteins in cells treated with LPS in the presence of various concentrations of esculetin were determined by western blot analysis. As shown in Fig. 2C, RAW 264.7 cells expressed high levels of iNOS and COX-2 proteins when stimulated with LPS alone; however, pretreatment with esculetin downregulated the expression of these LPS-stimulated proteins in a concentration-dependent manner. The results suggest that esculetin-induced reductions in the expression of iNOS and COX-2 were the cause of the inhibition of $\mathrm{NO}$ and $\mathrm{PGE}_{2}$ production.

Esculetin inhibits the production and expression of pro-inflammatory cytokines. We next attempted to determine the potential effects of esculetin on the LPS-induced release of pro-inflammatory cytokines, including TNF- $\alpha$ and IL-1 $\beta$, using ELISA. The results revealed that the levels of the two cytokines were increased in the culture media of LPS-stimulated RAW 264.7 cells, and these increases were significantly decreased in a concentration-dependent manner by pre-treatment with esculetin (Fig. 3A and B). In a parallel experiment, immunoblotting was performed to determine whether esculetin inhibited the expression of these cytokines.
From the results, it was observed that esculetin decreased the levels of TNF- $\alpha$ and IL-1 $\beta$ proteins in LPS-stimulated cells in a concentration-dependent manner (Fig. 3C). These results also indicate that esculetin acts primarily by preventing the accumulation of pro-inflammatory cytokines through alteration of their expression.

Esculetin inhibits LPS-induced nuclear translocation of $N F-\kappa B$ and degradation of $I \kappa B-\alpha$ in RAW 264.7 macrophages. Since NF- $\kappa \mathrm{B}$ is a central transcription factor that regulates the expression of a large number of inflammatory-related genes, we next examined the effect of esculetin on LPS-induced $\mathrm{NF}-\kappa \mathrm{B}$ translocation from the cytosol to the nucleus and I $\kappa \mathrm{B}-\alpha$ degradation. As shown in Fig. 4, the amount of NF- $\mathrm{B}$ p65 subunit in the nucleus was markedly increased upon exposure to LPS alone, but esculetin inhibited LPS-mediated nuclear translocation of $\mathrm{NF}-\kappa \mathrm{B}$ p 65 in a time-dependent manner. Under the same conditions, esculetin inhibited LPS-mediated IкB- $\alpha$ degradation, suggesting that esculetin may block LPS-induced nuclear translocation of NF- $\kappa$ B p65 by suppression of I $\kappa$ B- $\alpha$ degradation.

Esculetin inhibits the generation of ROS in LPS-stimulated $R A W 264.7$ macrophages. Since previous studies have demonstrated that LPS-induced ROS generation is associated with the activation of $\mathrm{NF}-\kappa \mathrm{B}$ (11), we examined the intracellular levels of ROS in order to determine whether intracellular redox states are involved in the inhibition of $\mathrm{NF}-\kappa \mathrm{B}$ signaling by esculetin. As shown in Fig. 5A, the levels of ROS were rapidly increased upon exposure to LPS alone; 

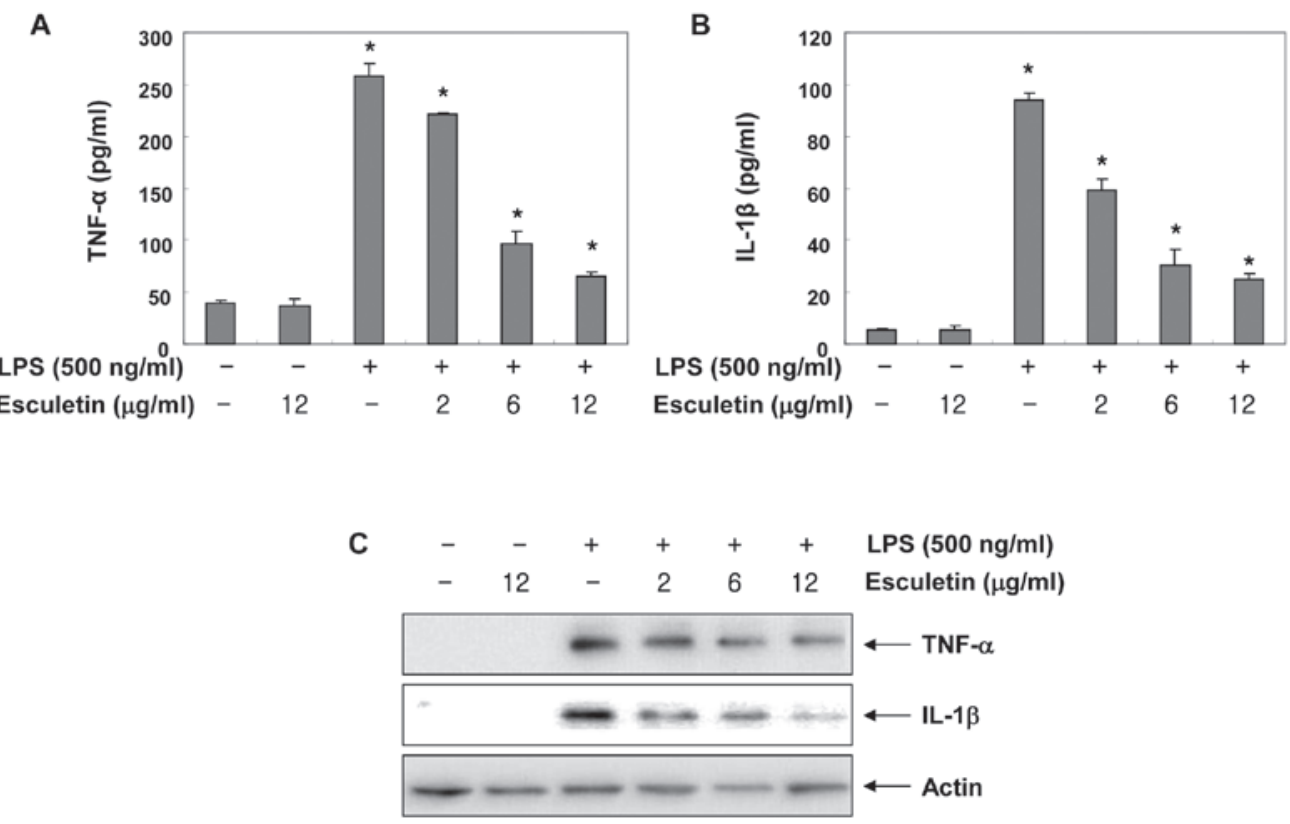

Figure 3. Effects of esculetin on production and expression of pro-inflammatory cytokines tumor necrosis factor- $\alpha$ (TNF- $\alpha$ ) and interleukin- $1 \beta$ (IL-1 $\beta$ ) in lipopolysaccharide (LPS)-challenged RAW 264.7 macrophages. RAW 264.7 cells were pre-treated with the indicated concentrations of esculetin for $1 \mathrm{~h}$ prior to LPS treatment (500 ng/ml). Following incubation for $24 \mathrm{~h}$, the levels of TNF- $\alpha$ (A) and IL-1 $\beta$ (B) present in the supernatants were measured. Each value indicates the mean \pm standard deviation and is representative of results obtained from three independent experiments. "P $<0.05$ compared with cells treated with LPS in the absence of esculetin. (C) Total proteins were isolated and subjected to SDS-polyacrylamide gel electrophoresis followed by western blot analysis using anti-TNF- $\alpha$ and anti-IL-1 $\beta$ antibodies, and an enhanced chemiluminescence detection system. Actin was used as an internal control.

A

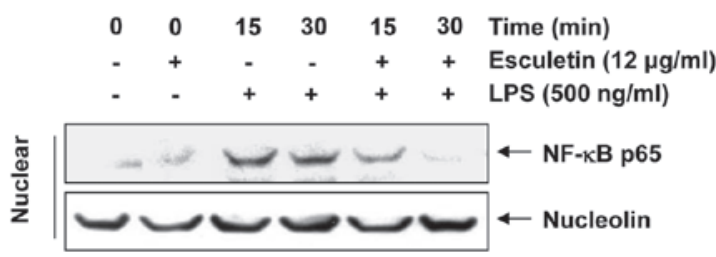

B

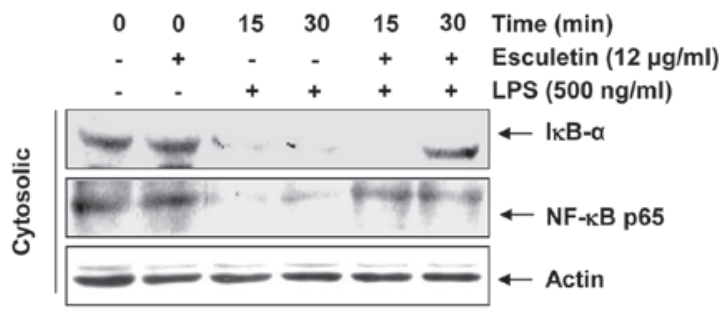

Figure 4. Effects of esculetin on lipopolysaccharide (LPS)-induced nuclear translocation of nuclear factor-kappa B (NF- $\mathrm{kB})$ and degradation of inhibitor

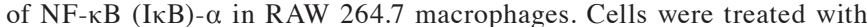
$12 \mu \mathrm{g} / \mathrm{ml}$ esculetin for $1 \mathrm{~h}$ prior to LPS treatment $(500 \mathrm{ng} / \mathrm{ml})$ for the indicated times. Nuclear (A) and cytosolic (B) proteins were subjected to $10 \%$ SDS-polyacrylamide gel electrophoresis followed by western blot analysis

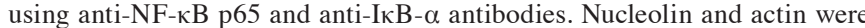
used as internal controls for the nuclear and cytosolic fractions, respectively.

however, pre-incubation with esculetin for $30 \mathrm{~min}$ attenuated the ROS generation in a dose-dependent manner in the LPS-activated RAW 264.7 macrophages (Fig. 5A). These results suggest that the inhibitory effects of esculetin on the $\mathrm{NF}-\kappa \mathrm{B}$ signaling pathway are at least partially correlated with a decrease in ROS generation. To examine whether esculetin is cytotoxic to RAW 264.7 cells, the cells were exposed to various concentrations of 7,8-DHF for $24 \mathrm{~h}$ in the presence or absence of LPS, and cell viability was then measured by the MTT assay. Within our tested concentrations, no cytotoxic effect of esculetin was observed in RAW 264.7 cells (Fig. 5B). These results clearly indicated that anti-inflammatory activity of esculetin in LPS-stimulated RAW 264.7 macrophages was not due to the cytotoxic action of esculetin.

\section{Discussion}

Coumarins including esculetin, fraxetin and daphnetin are fragrant organic chemical compounds in the benzopyrone chemical class, and are found naturally in a number of medicinal plants. Among them, esculetin is well known as a potent non-competitive inhibitor of lipoxygenase (24). Although this compound has multiple beneficial effects including anti-oxidant activity (15-17), to date, there is no data to demonstrate the antiinflammatory effects of esculetin in activated macrophages. In this study, we examined the effects of esculetin on the release of several pro-inflammatory mediators and cytokines as well as on ROS generation in LPS-stimulated murine macrophage RAW 264.7 cells. It was demonstrated that esculetin inhibited the expression of iNOS and COX-2 as well as NO and $\mathrm{PGE}_{2}$ production in LPS-treated RAW 264.7 macrophages. Esculetin also attenuated LPS-induced production and expression of pro-inflammatory cytokines including TNF- $\alpha$ and IL-1 $\beta$ as well as the generation of ROS, and these effects are mediated through the inhibition of nuclear translocation of $\mathrm{NF}-\kappa \mathrm{B}$.

Accumulated evidence indicates that $\mathrm{NO}$ and $\mathrm{PGE}_{2}$ are significant mediators of inflammation. Although three distinct isoforms of NOS have been isolated and cloned, a number of cell types express iNOS in their function in the host defense against microbial and viral pathogens, and this leads to the 

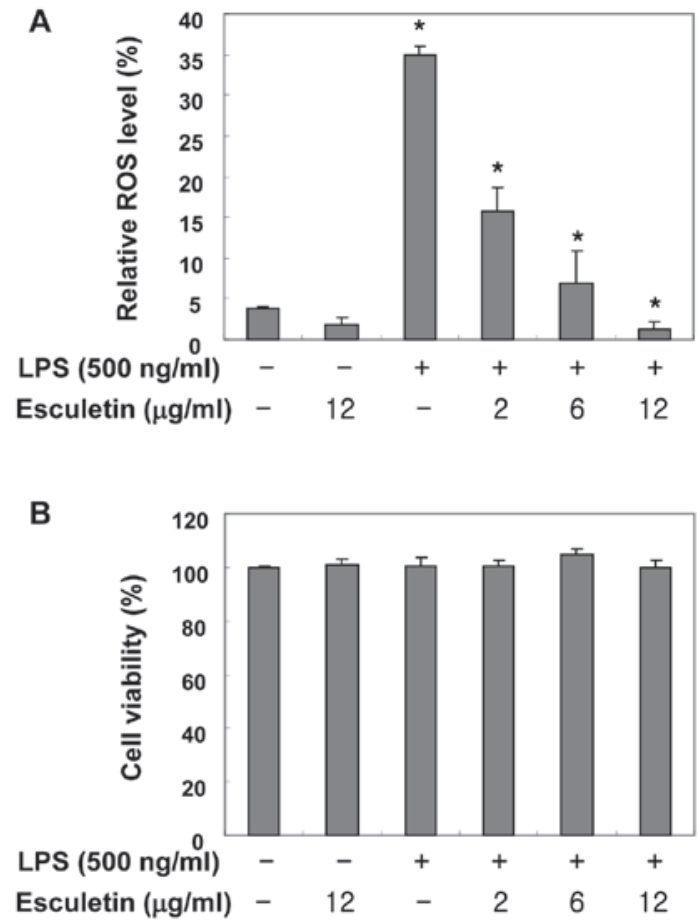

Figure 5. Effect of esculetin on lipopolysaccharide (LPS)-induced reactive oxygen species (ROS) generation and cell viability in RAW 264.7 macrophages. (A) Cells were pre-treated with the indicated concentrations of esculetin for $1 \mathrm{~h}$, then LPS $(500 \mathrm{ng} / \mathrm{ml})$ was added and cells were incubated for $30 \mathrm{~min}$. ROS generation was assessed using a ROS-sensitive fluorescence indicator, DCFH-DA, and flow cytometry. (B) Cells were treated with the indicated concentrations of esculetin or LPS alone, or pre-treated with various concentrations of esculetin for $1 \mathrm{~h}$ prior to LPS treatment. After $24 \mathrm{~h}$, cell viability was assessed using MTT reduction assays. The values are expressed as the mean \pm standard deviation from three experiments. ${ }^{*} \mathrm{P}<0.05$ compared with unstimulated control group.

formation of NO radicals. As a result, abnormally high levels of NO may contribute to the pathogenesis of inflammatory diseases $(25,26)$. COXs are enzymes that catalyze the conversion of arachidonic acid to $\mathrm{PGH}_{2}$, a precursor for a variety of biologically active mediators, including $\mathrm{PGE}_{2}$, prostacyclin and thromboxane A2. Of the two COX isoforms, $\mathrm{COX}-2$ is induced in response to numerous stimulants and is activated at sites of inflammation $(27,28)$. Therefore, endotoxin-induced NO and $\mathrm{PGE}_{2}$ production may be used as a measure of the progression of inflammation, and inhibition of their production may have potential therapeutic value for preventing inflammatory reaction and disease. In addition, it is well known that iNOS and COX-2 are rapidly and highly expressed in LPS-activated macrophages and contribute to the pathogenesis of septic shock $(29,30)$. In this study, we observed that esculetin inhibits LPS-induced NO and $\mathrm{PGE}_{2}$ synthesis in a concentrationdependent manner in RAW 264.7 macrophages. Consistently, this suppression was correlated with the downregulation of iNOS and COX-2 expression (Fig. 2).

Conversely, it has been reported that the dysregulation of cytokines including TNF- $\alpha$ and IL- $1 \beta$, commonly released by macrophages, plays an essential role in a number of undesirable inflammatory lesions. The production of these cytokines by activated macrophages is rapidly increased in acute inflammatory responses associated with infection, injury, trauma and other stress $(31,32)$. This production is then further increased by autocrine and paracrine routes, greatly increasing the severity of the immune response which causes inflammation $(33,34)$. Moreover, the production of TNF- $\alpha$ and IL-1 $\beta$ is crucially required for the synergistic induction of NO and $\mathrm{PGE}_{2}$ production in LPS-stimulated macrophages $(35,36)$. Thus, the selective inhibition of cytokine production and function may be effective therapeutically in the control of inflammatory disorders. In the present study, our data indicated that esculetin significantly inhibited the production of TNF- $\alpha$ as well as IL-1 $\beta$, which was in parallel with the comparable inhibition of their expression in RAW 264.7 cells stimulated by LPS (Fig. 3). In conjunction with inhibiting LPS-induced $\mathrm{NO}$ and $\mathrm{PGE}_{2}$ production, these findings provide evidence that esculetin possesses useful anti-inflammatory activity.

$\mathrm{NF}-\kappa \mathrm{B}$ transcription factor has been proven to play a key role in inflammatory responses through the regulation of genes encoding various inflammatory cytokines and inducible enzymes including COX-2 and iNOS in macrophages $(6,7,37)$. $\mathrm{NF}-\kappa \mathrm{B}$ is normally present in the cytosol due to its association with I $\mathrm{B}$. Once activated by inflammatory stimulants, I $\mathrm{B}$ is rapidly phosphorylated and degraded by the $26 \mathrm{~S}$ proteasome. IkB degradation liberates $\mathrm{NF}-\kappa \mathrm{B}$, which then translocates to the nucleus, resulting in transcriptional induction of inflammation-associated genes $(10,38,39)$. This study indicates that esculetin strongly inhibited the nuclear translocation of NF- $\kappa \mathrm{B}$ and blocked the degradation of I $\mathrm{B}$ in LPS-stimulated RAW 264.7 macrophages (Fig. 4). These observations suggest that the novel anti-inflammatory mechanisms mediated by esculetin are based on the inhibition of the LPS-mediated activation of $\mathrm{NF}-\kappa \mathrm{B}$.

Oxidative stress occurs when the balance of the intracellular redox state is disrupted by the excessive production of ROS. Accumulating evidence indicates that antioxidant compounds inhibit the production of inflammatory cytokines by suppressing the expression of the corresponding genes by suppressing $\mathrm{NF}-\kappa \mathrm{B}$ activation through the removal of ROS $(11,40,41)$. The suppressive effects of these compounds on the production of the associated inflammatory mediators are also associated with their antioxidant activities, and thus reduce inflammation $(2,42)$. Therefore, ROS is thought to be involved in inflammatory gene expression through the redoxbased activation of the NF- $\kappa \mathrm{B}$ signaling pathway. In this study, our data demonstrated that ROS production was markedly increased in LPS-stimulated RAW 264.7 cells and treatment with esculetin attenuated ROS production in a concentrationdependent manner (Fig. 5). These results indicate that esculetin has intracellular radical scavenging activity in RAW 264.7 cells and suggest that the potential inhibition of ROS generation by esculetin is consistent with the inhibition of the expression of $\mathrm{NF}-\kappa \mathrm{B}-$ dependent cytokines and mediators.

In summary, we have demonstrated that the treatment of mouse macrophage RAW 264.7 cells with esculetin decreased the levels of pro-inflammatory mediators including $\mathrm{NO}$ and $\mathrm{PGE}_{2}$ following LPS stimulation through downregulation of iNOS and COX-2 expression. Esculetin also significantly inhibited the formation of pro-inflammatory cytokines, including TNF- $\alpha$ and IL-1 $\beta$, which was associated with the suppression of expression of their corresponding genes. In addition, the anti-inflammatory properties of esculetin were mediated by the downregulation of $\mathrm{NF}-\kappa \mathrm{B}$ activation and the 
inhibition of ROS accumulation. Although further in vivo investigation is necessary to qualify the anti-inflammatory activity of esculetin, our data suggest that esculetin has a strong antioxidant capacity to inhibit inflammation-associated gene expression by suppressing the redox-dependent NF- $\mathrm{\kappa B}$ signaling pathway.

\section{Acknowledgements}

This study was supported by the Basic Science Research Program through the National Research Foundation of Korea (NRF) grant funded by the Korean government (no. 2012046358) and the R\&D program of MOTIE/KIAT (10040391, Development of Functional Food Materials and Device for Prevention of Aging-Associated Muscle Function Decrease), Republic of Korea.

\section{References}

1. Vendramini-Costa DB and Carvalho JE: Molecular link mechanisms between inflammation and cancer. Curr Pharm Des 18 3831-3852, 2012

2. Ma A, Qi S and Chen H: Antioxidant therapy for prevention of inflammation, ischemic reperfusion injuries and allograft rejection. Cardiovasc Hematol Agents Med Chem 6: 20-43, 2008

3. Carreira PE: Pulmonary hypertension in autoimmune rheumatic diseases. Autoimmun Rev 313-320, 2004.

4. Fialkow L, Wang Y and Downey GP: Reactive oxygen and nitrogen species as signaling molecules regulating neutrophil function. Free Radic Biol Med 42: 153-164, 2007.

5. Geronikaki AA and Gavalas AM: Antioxidants and inflammatory disease: synthetic and natural antioxidants with anti-inflammatory activity. Comb Chem High Throughput Screen 9: 425-442, 2006.

6. Gasparini C and Feldmann M: NF- $\kappa \mathrm{B}$ as a target for modulating inflammatory responses. Curr Pharm Des 18: 5735-5745, 2012.

7. Lawrence T: The nuclear factor NF-kappaB pathway in inflammation. Cold Spring Harb Perspect Biol 1: a001651, 2009.

8. Siomek A: NF- $\kappa \mathrm{B}$ signaling pathway and free radical impact. Acta Biochim Pol 59: 323-331, 2012.

9. Temkin V and Karin M: From death receptor to reactive oxygen species and c-Jun N-terminal protein kinase: the receptorinteracting protein 1 odyssey. Immunol Rev 220: 8-21, 2007.

10. Morgan MJ and Liu ZG: Crosstalk of reactive oxygen species and NF- $\kappa$ B signaling. Cell Res 21: 103-115, 2011.

11. Gloire G, Legrand-Poels S and Piette J: NF-kappaB activation by reactive oxygen species: fifteen years later. Biochem Pharmacol 72: 1493-1505, 2006.

12. Fylaktakidou KC, Hadjipavlou-Litina DJ, Litinas KE and Nicolaides DN: Natural and synthetic coumarin derivatives with anti-inflammatory/antioxidant activities. Curr Pharm Des 10: 3813-3833, 2004.

13. Witaicenis A, Seito LN and Di Stasi LC: Intestinal antiinflammatory activity of esculetin and 4-methylesculetin in the trinitrobenzenesulphonic acid model of rat colitis. Chem Biol Interact 186: 211-218, 2010

14. Kwon OS, Choi JS, Islam MN, Kim YS and Kim HP: Inhibition of 5-lipoxygenase and skin inflammation by the aerial parts of Artemisia capillaris and its constituents. Arch Pharm Res 34: 1561-1569, 2011.

15. Prabakaran D and Ashokkumar N: Protective effect of esculetin on hyperglycemia-mediated oxidative damage in the hepatic and renal tissues of experimental diabetic rats. Biochimie 95: 366-373, 2013.

16. Subramaniam SR and Ellis EM: Esculetin-induced protection of human hepatoma HepG2 cells against hydrogen peroxide is associated with the Nrf2-dependent induction of the $\mathrm{NAD}(\mathrm{P}) \mathrm{H}$ : Quinone oxidoreductase 1 gene. Toxicol Appl Pharmacol 250: 130-136, 2011.

17. Kim SH, Kang KA, Zhang R, et al: Protective effect of esculetin against oxidative stress-induced cell damage via scavenging reactive oxygen species. Acta Pharmacol Sin 29: 1319-1326, 2008.
18. Lee CR, Shin EJ, Kim HC, et al: Esculetin inhibits N-methyl-Daspartate neurotoxicity via glutathione preservation in primary cortical cultures. Lab Anim Res 27: 259-263, 2011.

19. Leung KN, Leung PY, Kong LP and Leung PK: Immunomodulatory effects of esculetin (6,7-dihydroxycoumarin) on murine lymphocytes and peritoneal macrophages. Cell Mol Immunol 2: 181-188, 2005.

20. Park C, Jin CY, Kim GY, et al: Induction of apoptosis by esculetin in human leukemia U937 cells through activation of JNK and ERK. Toxicol Appl Pharmacol 227: 219-228, 2008.

21. Park C, Jin CY, Kwon HJ, et al: Induction of apoptosis by esculetin in human leukemia U937 cells: roles of Bcl-2 and extracellular-regulated kinase signaling. Toxicol In Vitro 24: 486-494, 2010.

22. Kyung J, Kim D, Park D, et al: Synergistic anti-inflammatory effects of Laminaria japonica fucoidan and Cistanche tubulosa extract. Lab Anim Res 28: 91-97, 2012.

23. Lee SH, Kim DW, Eom SA, et al: Suppression of 12-O-tetradecanoylphorbol-13-acetate (TPA)-induced skin inflammation in mice by transduced Tat-Annexin protein. BMB Rep 45: 354-359, 2012.

24. Sekiya K, Okuda H and Arichi S: Selective inhibition of platelet lipoxygenase by esculetin. Biochim Biophys Acta 713: 68-72, 1982.

25. Yang CS, Yuk JM and Jo EK: The role of nitric oxide in mycobacterial infections. Immune Netw 9: 46-52, 2009.

26. Rimoldi JM and Chimote SS: Inhibition on inducible nitric oxide synthase. Curr Opin Drug Discov Devel 1: 183-191, 1998.

27. Murakami A and Ohigashi H: Targeting NOX, INOS and COX-2 in inflammatory cells: chemoprevention using food phytochemicals. Int J Cancer 121: 2357-2363, 2007.

28. Wadleigh DJ, Reddy ST, Kopp E, Ghosh S and Herschman HR: Transcriptional activation of the cyclooxygenase-2 gene in endotoxin-treated RAW 264.7 macrophages. J Biol Chem 275: 6259-6266, 2000.

29. Kanno S, Shouji A, Tomizawa A, et al: Inhibitory effect of naringin on lipopolysaccharide (LPS)-induced endotoxin shock in mice and nitric oxide production in RAW 264.7 macrophages. Life Sci 78: 673-681, 2006.

30. Lin WJ and Yeh WC: Implication of Toll-like receptor and tumor necrosis factor alpha signaling in septic shock. Shock 24 : 206-209, 2005

31. Dayer JM: The process of identifying and understanding cytokines: from basic studies to treating rheumatic diseases. Best Pract Res Clin Rheumatol 18: 31-45, 2004.

32. Guha M and Mackman N: LPS induction of gene expression in human monocytes. Cell Signal 13: 85-94, 2001.

33. Pearlman DS: Pathophysiology of the inflammatory response. J Allergy Clin Immunol 104: S132-S137, 1999.

34. Beutler B and Cerami A: The biology of cachectin/TNF- $\alpha$ primary mediator of the host response. Annu Rev Immunol 7: 625-655, 1989.

35. Blatteis CM, Li S, Li Z, Perlik V and Feleder C: Signaling the brain in systemic inflammation: the role of complement. Front Biosci 9: 915-931, 2004.

36. Aggarwal BB and Natarajan K: Tumor necrosis factors: developments during the last decade. Eur Cytokine Netw 7: 93-124, 1996.

37. Moynagh PN: The NF-kappaB pathway. J Cell Sci 118: 4589-4592, 2005.

38. Sarkar FH, Li Y, Wang Z and Kong D: NF-kappaB signaling pathway and its therapeutic implications in human diseases. Int Rev Immunol 27: 293-319, 2008.

39. Makarov SS: NF-kappaB as a therapeutic target in chronic inflammation: recent advances. Mol Med Today 6: 441-448, 2000.

40. Islam MN, Choi RJ, Jin SE, et al: Mechanism of antiinflammatory activity of umbelliferone 6-carboxylic acid isolated from Angelica decursiva. J Ethnopharmacol 144: 175-181, 2012.

41. Jung WK, Choi I, Lee DY, et al: Caffeic acid phenethyl ester protects mice from lethal endotoxin shock and inhibits lipopolysaccharide-induced cyclooxygenase- 2 and inducible nitric oxide synthase expression in RAW 264.7 macrophages via the p38/ERK and NF-kappaB pathways. Int J Biochem Cell Biol 40: 2572-2582, 2008

42. Harris J, Olière S, Sharma $S$, et al: Nuclear accumulation of cRel following C-terminal phosphorylation by TBK1/IKK epsilon. J Immunol 177: 2527-2535, 2006. 